\title{
A rare case of cervical ectopic: potential life-threatening condition managed conservatively with uterine artery embolisation and Methotrexate
}

\author{
Joyita Bhowmik, Amit Kyal*, Aprateem Mukherjee, Indrani Das, \\ Vidhika Berwal, Pijush Kanti Das
}

Department of Obstetrics and Gynecology, Medical College, Kolkata, 88 College Street, West Bengal, India

Received: 23 February 2018

Accepted: 28 March 2018

*Correspondence:

Dr. Amit Kyal,

E-mail: amitkyal@yahoo.com

Copyright: ( $)$ the author(s), publisher and licensee Medip Academy. This is an open-access article distributed under the terms of the Creative Commons Attribution Non-Commercial License, which permits unrestricted non-commercial use, distribution, and reproduction in any medium, provided the original work is properly cited.

\begin{abstract}
A case of cervical pregnancy managed successfully in Medical College, Kolkata by injection Methotrexate and uterine artery embolization. Cervix is a rare implantation site for ectopic pregnancy. Either during surgical management should be carefully considered due to the risk of severe hemorrhage. A 33 years old patient (P2+4 with 1 living issue) with USG diagnosed 6 weeks cervical pregnancy was admitted in Gynaecology and Obstetrics department of Medical College, Kolkata with slight bleeding per vaginum and pain abdomen. USG was repeated along with beta hCG quantification and other routine investigations. Following admission, the bleeding and pain subsided. Patient was counselled regarding the prognosis and management options available. The patient was desirous to preserve her fertility and as the patient was hemodynamically stable with low initial beta hCG of $5200 \mathrm{mIU} / \mathrm{ml}$, we opted for a medical treatment with MTX and uterine artery embolization. Following treatment with 3 doses of weekly Methotrexate $\left(50 \mathrm{mg} / \mathrm{m}^{2}\right.$ each i.m.) and UAE the beta hCG reduced significantly. The patient was discharged with advice of weekly follow up with beta hCG report. Within 2 months of follow-up, B-hCG level was $<10 \mathrm{mIU} / \mathrm{mL}$ with TVS showing normal cervical canal and empty uterine cavity.
\end{abstract}

Keywords: Cervical pregnancy, Fertility, Methotrexate, Uterine artery embolization

\section{INTRODUCTION}

Cervical ectopic pregnancy (CEP) is a rare form of ectopic pregnancy which is often associated with significant morbidity and devastating effects on future fertility. ${ }^{1,2}$ It accounts for $<1 \%$ of all ectopic gestations. Its incidence varies between 1 in 2500 to 18000 pregnancies. ${ }^{1,2}$ Known risk factors for CEP include procedures or pathology that result in cervical scarring or injury, such as cesarean section, curettage, cervical conization, or Asherman's syndrome. ${ }^{3,4}$ In vitro fertilization has also been associated with an increased incidence of CEP. Treatment of cervical pregnancy is a therapeutic dilemma. Surgical treatment is often associated with a risk of torrential haemorrhage resulting in hysterectomy and even maternal death.

Therefore, in an attempt to avoid hysterectomy and preserve fertility, a more conservative therapeutic approach is being developed: chemotherapy, cerclage, foleys catheter tamponade, hypogastric iliac artery ligation, and uterine artery embolization under angiographic control., ${ }^{5,6}$ The advent of first-trimester ultrasound has led to improvements in the early diagnosis 
of CEP. UAE is a potentially fertility sparing technique for CEP management. ${ }^{7,8}$

In this report, we present a stepwise conservative approach using uterine artery embolization in conjunction with injection methotrexate for cervical pregnancy.

\section{CASE REPORT}

A 33-year-old $7^{\text {th }}$ gravida $\mathrm{P}_{2+4}$ with previous two caesarean sections and only 1 living issue was referred to our institute with USG diagnosed cervical ectopic pregnancy of 6 weeks gestation.

On admission her vitals were stable but had few episodes of slight bleeding per vagina and pain abdomen. Her medical history was unremarkable. She underwent hysteroscopy in 2017 for the evaluation of secondary infertility which revealed uterine synechia which was dissected. Pelvic examination on admission revealed a barrel-shape cervix with minimal bleeding. Again, TVS was done in our institute which confirmed the presence of gestational sac in the anterior wall of cervix at the level of internal os with vascularity in doppler study (Figure 1).

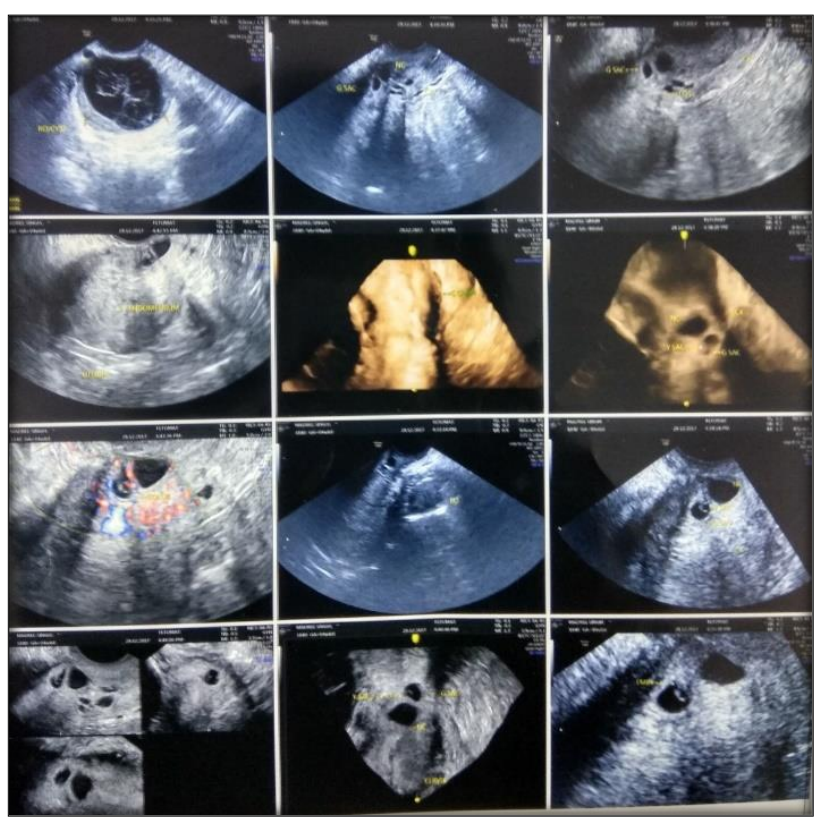

Figure 1: TVS showing gestational sac present in cervix.

Her initial beta hCG was $5200 \mathrm{mIU} / \mathrm{L}$. Patient was offered various options (medical and surgical) available but she declined surgical intervention with desire to preserve her fertility. So, decision in favour of medical management by giving injection methotrexate i.m. at a dose of $50 \mathrm{mg} / \mathrm{m}^{2}$ along with UAE was taken. The first dose of injection MTX was given and beta hCG was repeated on day 4 and day 7 following MTX injection with a plan to repeat MTX if the decline in beta HCG was less than $15 \%$. Meanwhile interventional radiologist of our institute was consulted to evaluate patient for UAE.
The potential risks of treatment were explained to her, written informed consent obtained and the procedure of UAE was done uneventfully (Figure 2 and 3).

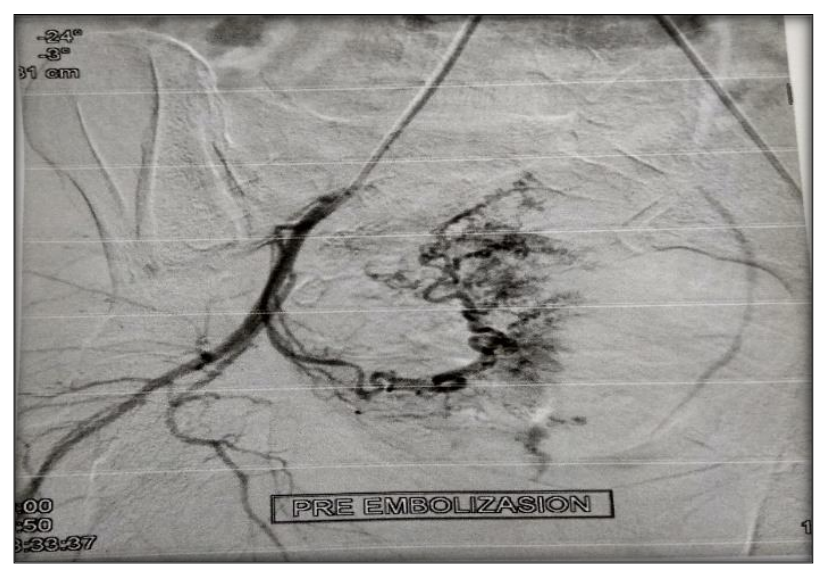

Figure 2: Pre-embolisation angiograph of uterine artery.

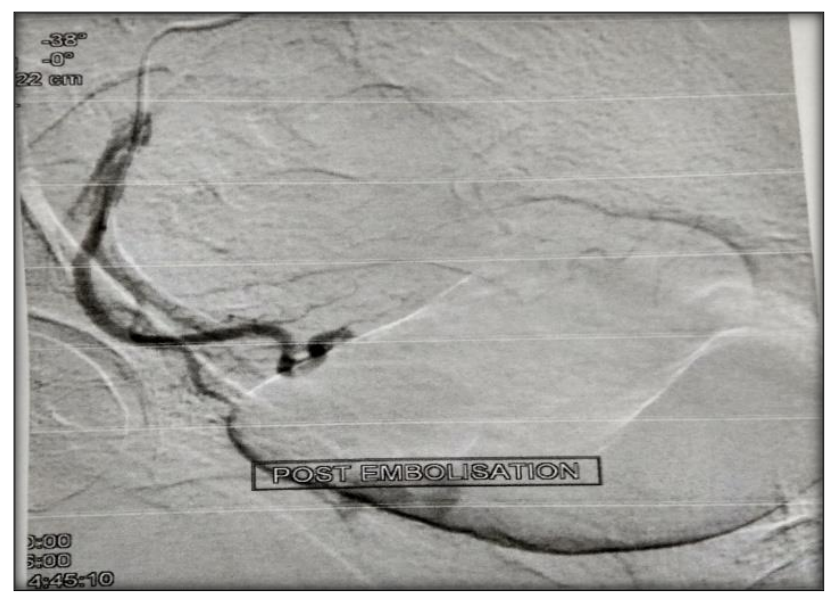

Figure 3: Post embolisation angiograph.

In total she received 3 doses of injection MTX. Subsequent beta hCG values showed declining trends (>15\%). Patient was discharged in stable condition with advice to follow up in the OPD at weekly intervals with beta HCG report. After 2 months follow-up, $\beta$-hCG level was $<10 \mathrm{mIU} / \mathrm{mL}$ with TVS showing normal cervical canal and empty uterine cavity.

This synergistic two-pronged approach of UAE in conjunction with systemic methotrexate administration has demonstrated high efficacy in this case in preserving fertility.

\section{DISCUSSION}

Cervical ectopic pregnancies have a low incidence ( 1 in 2,500-18,000 pregnancies), even when considering the most common extrauterine implantation sites, they roughly represent $1 \%$ of overall ectopic pregnancies. ${ }^{9,10}$ The anatomic site and complications associated to it, 
award special considerations due to significant hemorrhage risk. Specific causes remain unknown though previous conditions like fibroids, curettage, cesarean sections, Asherman's syndrome and intrauterine devices, all may alter implantation surface conditions increasing risk for an ectopic implantation. ${ }^{11,12}$

Assisted Reproduction Techniques (ART) is currently on the rise and associated to a $3.7 \%$ incidence of ectopic gestations, rendering higher possibilities for cervical pregnancies. ${ }^{13}$ Sonoghraphic criteria of cervical pregnancy are endocervical localization of gestational sac and trophoblastic invasion. A gestational sac can be visualized below the internal os and colour doppler examination can demonstrate peritrophoblastic blood flow characteristic of early implantation. Most patients with cervical ectopic pregnancy are women with low parity.

With today's more widespread application of arterial angiography in gynaecology and obstetrics, selective arterial embolization has become accepted as a highly effective technique for controlling acute and chronic genital bleeding (Chin et al; Vedantham et al). Thus, the current treatment is to preserve reproductive functions. Systemic or intraamniotic MTX administration, intrasac KCL injection, vasopressin infiltration, vacuum evacuation of cervical canal followed by balloon tamponade with a Foley's catheter are some of the treatment techniques. The main problem with management of cervical pregnancy is the possibility of a life-threatening haemorrhage before or after pregnancy evacuation. The successful use of UAE to obviate hysterectomy in the treatment of cervical ectopic pregnancy when fertility is desired has been reported. The procedure was used by Cosin et al to avoid surgery in a patient with a cervical pregnancy. ${ }^{17}$ Nomiyama et al reported on a conservative treatment using preventive selective uterine artery embolization, followed by intraamniotic MTX instillation. ${ }^{16}$ Risks of embolotherapy include uterine infarction or ischemia and necrosis, however, the majority tolerate the procedure well. ${ }^{14,15}$ The rationale for combining the two techniques was based not only on the expected difficulties but also on the fact that UAE may prevent possible uterine hemorrhages caused by the injection of MTX.

This dual approach of UAE in conjunction with systemic methotrexate is an emerging best mode of management which reduces the risk of life threatening haemorrhage and preservation of fertility. ${ }^{18}$

\section{CONCLUSION}

Cervical pregnancy is an uncommon but potentially lifethreatening entity. UAE along with systemic methotrexate is a safe, minimally invasive and effective procedure with low rate of complications for cervical ectopic pregnancy. A fertility sparing approach is the optimal treatment for those patient population which has higher rates of infertility and nulliparity.

Funding: No funding sources

Conflict of interest: None declared

Ethical approval: Not required

\section{REFERENCES}

1. Parente JT, Ou CS, Levy J, Legatt E. Cervical pregnancy analysis: a review and report of five cases. Obstet Gynecol 1983;62:79-82.

2. Dees HC. Cervical pregnancy associated with uterine leiomyomas. South Med J. 1966;59:900-5.

3. Bayati J, Garcia JE, Dorsey JH, Padilla SL. Combined intrauterine and cervical pregnancy from in vitro fertilization and embryo transfer. Fertil Steril. 1989;51:725-7.

4. Bennett S, Waterstone J, Parsons J, Creighton S. Two cases of cervical pregnancy following in vitro fertilization and embryo transfer to the lower uterine cavity. J Assist Reprod Genet. 1993;10:100-3.

5. Ratten GJ. Cervical pregnancy treated by ligation of the descending brance of the uterine arteries: case report. Br J Obstet Gynaecol. 1983;90:367-71.

6. Cervical pregnancy managed by placement of a Shirodkar cerclage before evacuation: a case report. J Repro Med. 1989;34:69-70.

7. Pattinson HA, Dunphy BC, Wood S, Saliken J. Cervical pregnancy following in vitro fertilization: evacuation after uterine artery embolization with subsequent successful intrauterine pregnancy. Aust N Z J Obstet Gynaecol. 1994;34:492-3.

8. Gun M, Mavrogiorgis M. Cervical ectopic pregnancy: a case report and literature review. Ultrasound Obstet Gynecol. 2002;19:297-301.

9. Ushakov FB, Elchalal U, Aceman PJ, Schenker JG. Cervical pregnancy: past and future. Obstet Gynecol Surv. 1997;52:45-59.

10. Verma U, Goharkhay N. Conservative management of cervical ectopic pregnancy. Fertil Steril. 2009;91:671-4.

11. Fylstra DL. Cervical pregnancy: 13 cases treated with suction curettage and balloon tamponade. Am J Obstet Gynecol. 2014;210:581.

12. Mohebbi MR, Rosenkrans KA, Luebbert EE, Hunt TT, Jung MJ. Ectopic pregnancy in the cervix: a case report. Case Rep Med. 2011:858241.

13. Hulvert J, Mardesic T, Voboril J, Müller $P$. Heterotopic pregnancy and its occurrence in assisted reproduction. Ceska Gynekol. 1999;64:299-301.

14. Godfrey CD, Zbella EA. Uterine necrosis after uterine artery embolization for leiomyoma. Obstet Gynecol 2001;98:950-2.

15. Pron G, Bennett J, Common A, Sniderman K, Asch $\mathrm{M}$, Bell S, et al. Technical results and effects of operator experience on uterine artery embolization for fibroids: the ontario uterine fibroid embolization Trial. J Vasc Interv Radiol. 2003;14:545-54. 
16. Nomiyama M, Arima K, Iwasaka T. Conservative treatment using a methotrexate-lipidol emulsion containing non-ionic contrast medium for a cervical ectopic pregnancy. Hum Reprod. 1997;12:2826-9.

17. Cosin JA, Bean M, Grow D. et al The use of methotrexate and arterial emblization to avoid surgery in a case of cervical pregnancy. Fertil Steril. 1997;67:1169-71.
18. Radhakrishna KP, Pushpalatha LV,et al. Role of uterine artery embolization and methotrexate in cervical pregnancy. JSAFOMS. 2014;2(1):35-7

Cite this article as: Bhowmik J, Kyal A, Mukherjee A, Das I, Berwal V, Das PK. A rare case of cervical ectopic: potential life-threatening condition managed conservatively with uterine artery embolisation and Methotrexate. Int J Reprod Contracept Obstet Gynecol 2018;7:2061-4. 